\title{
CLINICAL PROFILE AND IN-HOSPITAL OUTCOME OF PATIENTS WITH RIGHT VENTRICULAR MYOCARDIAL INFARCTION IN INFERIOR WALL MYOCARDIAL INFARCTION
}

\author{
Ravi Prakash Pandey1, Kuwer Devi Singh², Mohsin Bilal ${ }^{3}$, Manzar Hussain Usmani ${ }^{4}$ \\ ${ }^{1}$ Assistant Professor, Department of Medicine, SS Medical College and SGM Hospital, Rewa. \\ ${ }^{2}$ Assistant Professor, Department of Cardiology, SS Medical College and SGM Hospital, Rewa. \\ ${ }^{3}$ Postgraduate Student, Department of Medicine, SS Medical College and SGM Hospital, Rewa. \\ ${ }^{4}$ Associate Professor, Department of Medicine, SS Medical College and SGM Hospital, Rewa.
}

\begin{abstract}
BACKGROUND

The present study deals with the clinical profile of right ventricular infarction as diagnosed by right precordial electrocardiography in patients with acute inferior wall myocardial infarction. Recognition of the syndrome of RVI is important, as it identifies a significant clinical entity which is associated with and is an independent predictor of major complications and in hospital mortality after acute IWMI. The main purpose of this study was to become aware of RVI and its complications like hypotension, arrhythmias and conduction disorders, which not only need appropriate therapy but also requires avoidance of inappropriate therapy, which may reduce right ventricular filling pressure and cardiac output further worsening the condition.

Aims and Objective - The study emphasises on the incidence of RVMI in patients of acute inferior wall MI using right precordial ECG, the clinical profile of patients with RVMI in acute IWMI and to study the incidence of complications in patients of acute IWMI.
\end{abstract}

\section{MATERIALS AND METHODS}

This is a prospective study carried out on patients admitted with inferior wall MI in our tertiary care hospital from March 2015 to May 2016. At the time of admission, a seventeen lead ECG consisting of twelve conventional leads and additional right precordial leads V3R, V4R, V5R, V6R was taken and subsequent recordings were made for conventional and right precordial leads.

\section{RESULTS}

Out of 90 patients of acute IWMI admitted to ICCU Department of Medicine, 25 patients were diagnosed with RVMI (28\%). Patients were continuously monitored during hospital stay and their serial ECGs clinical course and response to treatment were analysed. There was five times increased incidence of mortality in patients of IWMI with RVMI as compared to no RVMI. Complications like cardiogenic shock, various arrhythmia viz. AV block, VT were higher in RVMI group.

\section{CONCLUSION}

RVMI occurs in a significant number of patients with inferior wall MI. It can be easily diagnosed with considerable accuracy by a simple bedside routine 12-lead ECG along with right-sided ECG leads. Early diagnosis of RVMI can prevent fatal complications like high degree heart blocks, ventricular arrhythmias, cardiogenic shock and high in-hospital mortality. Patients who survive the acute phase have no long-term consequences and complete recovery over a period of weeks to months is the rule in majority. Therefore, right-sided ECG should be taken routinely in all patients of IWMI.

\section{KEYWORDS}

Inferior Wall Myocardial Infarction, Right Ventricular Infarction, Electrocardiography, Jugular Venous Pressure.

HOW TO CITE THIS ARTICLE: Pandey RP, Singh KD, Bilal M, et al. Clinical profile and in-hospital outcome of patients with right ventricular myocardial infarction in inferior wall myocardial infarction. J. Evolution Med. Dent. Sci. 2017;6(15):1193-1199, DOI: $10.14260 /$ Jemds/2017/260

\section{BACKGROUND}

Myocardial infarction was previously thought to be a disease of mainly the left ventricle. Right ventricular infarction was just a pathological entity. In 1974, Cohn ${ }^{1}$ for the first time described potentially serious and unique haemodynamic consequences of right ventricular infarction. The advent of more sophisticated diagnostic techniques and more precise

Financial or Other, Competing Interest: None.

Submission 15-01-2017, Peer Review 09-02-2017,

Acceptance 14-02-2017, Published 20-02-2017.

Corresponding Author:

Dr. Ravi Prakash Pandey,

F-12, Doctor's Colony,

Medical College Campus,

Rewa-486001, Madhya Pradesh.

E-mail: drraviprakashpandey@gmail.com

DOI: $10.14260 /$ jemds $/ 2017 / 260$ haemodynamic measurement has demonstrated that right ventricular infarction is a well-defined clinical entity and value of recognising patients with predominant right ventricular dysfunction is related not only to instituting appropriate therapy for severe pump failure, but also to avoid inappropriate therapy. It has also been shown that right ventricular infarction occurs most commonly in association with inferior myocardial infarction or inferoposterior myocardial infarction. ${ }^{2}$ Although, isolated right ventricular infarction had been described in autopsy reports as less than $3 \%$ of all acute myocardial infarction, ${ }^{2}$ the incidence of right ventricular infarction associated with IWMI has been shown to be as high as $30 \%-50 \% .3,4,5$

It results primarily from occlusion of the right coronary artery and infrequently from involvement of the left anterior descending artery and occasionally in inferoposterior left ventricular infarction also.2,4 
Right ventricular myocardial infarction can lead to diminished right-sided stroke volume with concomitant right ventricular dilatation and septal changes. The potential haemodynamic derangement associated with right ventricular infarction renders the patient's unusually sensitive to diminished ventricular preload. These two circumstances can result in a severe decrease in right and secondarily left ventricular output resulting in a clinical triad of hypotension and jugular venous pressure distension in the presence of clear lung fields. $6,7,8$

It is known that ST segment elevation of $0.1 \mathrm{mV}$ or greater in one or more of right precordial leads V4R to V6R is highly sensitive (90\%) and specific (91\%) in identifying acute right ventricular infarction. ${ }^{9,10}$

A subtle clue to the presence of haemodynamically significant right ventricular infarction is a marked sensitivity to preload reducing agents such as nitrates, morphine or diuretics. Prompt fluid therapy may abort the vicious cycle set in motion by right ventricular infarction, which if treated in conventional way or neglected tends to lead to true cardiogenic shock. ${ }^{11,12}$

Recognition of the syndrome of RVI is important, as it identifies a significant clinical entity, which is associated with and is an independent predictor of major complications and in-hospital mortality after acute inferior MI.12,13

It is associated with increased risk of death, shock, ventricular tachycardia or fibrillation and Atrioventricular Block (AVB) and a higher mortality rate for the first month post MI in patients with RVMI, but without haemodynamic impairment. These complications in RVMI may be due to the increased parasympathetic tone, Sinoatrial (SA) node dysfunction, Atrioventricular (AV) node dysfunction. $4,13,14$

Thus, the present study deals with clinical profile of right ventricular infarction as diagnosed by right precordial electrocardiography in patients with acute inferior myocardial infarction. Main purpose of selecting this study was to become aware of right ventricular infarction and its various complications like arrhythmias and conduction disorders, which not only requires appropriate therapy but also avoidance of inappropriate therapy that might reduce right ventricular filling pressure and cardiac output and in turn prove to be disastrous.

\section{Aims and Objective}

The present study titled incidence, clinical profile and inhospital outcome of patients of RVMI in IWMI was carried out in patients admitted in Department of Medicine, S. S. Medical College and Associated S. G. M. H, Rewa (M. P.) from March 2015 to May 2016 with the following aims and objectives -

1. To study the incidence of right ventricular infarction in patients of acute inferior myocardial infarction using right precordial electrocardiography.

2. To study the clinical profile of patients of RVMI in acute IWMI.

3. To study the incidence of complications in patients of acute IWMI with and without RVMI.

4. To compare the mortality in patients of IWMI with and without RVMI.

5. To study the response of specific therapy in patients with right ventricular infarction.

\section{MATERIALS AND METHODS}

This was a prospective clinical study conducted on 90 patients between March 2015 - May 2016 admitted in ICCU of our Tertiary Care Centre with IWMI.

At the time of admission, a seventeen lead ECG consisting of twelve conventional leads and additional right precordial leads V3R, V4R, V5R, V6R were taken and subsequent recordings made for conventional and right precordial leads. For recordings, a single channel ECG machine was used. All ECG's were recorded in $25 \mathrm{~mm} /$ second $10 \mathrm{mV}$ setting. The points on chest wall used for recording chest leads were marked with a skin pencil, so that same points could be used serially in a given patient. Serial ECG's were taken on admission, at the end of $6 \mathrm{~h}$, end of $12 \mathrm{~h}$ and daily till ST segment became isoelectric in right precordial leads. ECG's were also repeated whenever patient complained of chest pain. Patients were continuously monitored. The diagnosis of acute inferior wall myocardial infarction was made as typical history of chest pain, ST segment elevation in leads II, III and avF and by development of pathological $Q$ waves in the above mentioned leads. Tall ' $\mathrm{R}$ ' waves in V1 and V2 and increased serum cardiac enzymes Troponin- $T$ [qualitative]. The diagnosis of right ventricular myocardial infarction will be made as by the criteria of ST segment elevation of $0.1 \mathrm{mV}$ or more in one or more of the right precordial leads (V3R, V4R, V5R and V6R) in those patients who satisfied the criteria for an inferior wall myocardial infarction.

\section{Patients were classified into Two Groups}

Group A: Inferior wall infarction with right ventricular infarction.

Group B: Inferior wall infarction without right ventricular infarction.

Their clinical course were studied and compared. The clinical course and ECG analysis was compared in both the groups during their entire hospital stay. Complications viz. ventricular tachycardia, AV blocks, hypotension, cardiogenic shock and deaths in both the groups were analysed and compared.

\section{Inclusion Criteria}

All patients with definite evidence of acute inferior wall myocardial infarction as proved by 12-lead ECG along with right precordial leads and chest pain of duration less than 24 hours were considered in our study.

\section{Exclusion Criteria}

1. History of chest pain of more than $24 \mathrm{~h}$ duration.

2. Patients whose initial ECG's showed an anteroseptal or anterior wall myocardial infarction will be also excluded, because these infarctions may produce an anteriorly oriented ST vector which may also cause ST segment elevation in the right precordial leads. For the same reason patients with pericarditis, left bundle branch block were excluded.

3. Patients with chronic lung disease, cor pulmonale were also excluded because they may be associated with a right ventricular dysfunction.

4. Patients with previous history of a myocardial infarction were also excluded to avoid a false positive result for right precordial electrocardiography. 
Following Clinical Parameters were considered for Comparison viz.,

1. Hypotension was defined as systolic arterial blood pressure $<90 \mathrm{mmHg}$ or which has declined by at least $30 \mathrm{mmHg}$ below previous level.

2. Right heart failure was considered when the hepatojugular reflux or abdominal compression test will be positive. JVP more than $4 \mathrm{~cm}$ in $45^{\circ}$ position with pedal oedema and systemic venous congestion.

3. Ventricular tachycardia.

4. AV block $2^{\circ}$ and higher.

5. Death.

6. Cardiogenic shock

Left heart failure was considered when bilateral pulmonary rales was present on inspiration; dullness on percussion over lung bases and on auscultation S3 and S4 were present. Peripheral pulse shows pulsus alternans. Chest X-ray shows Kerley B lines. Present study deals with clinical profile of right ventricular infarction as diagnosed by right precordial electrocardiography in patients of acute inferior wall myocardial infarction. The results obtained were analysed for their statistical significance using Fisher's exact test and unpaired ' $\mathrm{t}$ ' test.

\section{RESULTS}

90 patients of acute inferior wall MI were admitted in ICCU, Department of Medicine during the study period from March 2015 to May 2016 and their serial 12-lead ECGs along with right-sided leads RV3, RV4 were taken at the time of admission, then every six hourly and thereafter whenever the patient complained of chest pain. Patients were continuously monitored during hospital stay and their serial ECGs, clinical course and response to the treatment was analysed.

Out of 90 patients admitted with IWMI, 25 patients had RVMI (28\%) and 65 patients were without RVMI (72\%) in IWMI (Table 1). Incidence of RVMI was more in females (36\%) as compared to males (22\%); $24 \%$ hypertensive patients had RV involvement, while in diabetics the incidence was $30 \%$. Tobacco and positive family history contributed significantly in IWMI with incidence being $40 \%$ and $34.4 \%$ respectively. Maximum number of patients presented with chest pain (100\%) followed by perspiration (33\%), dyspnoea (22\%), palpitations (17.77\%) and syncope (10\%). Raised JVP was an important sign in RVMI $(60 \%)$ as compared to patients without RVMI (30\%) (Table 2). There was five times increase in mortality in patients of IWMI with RVMI (16\%) as compared to (3\%) in no RVMI group (Table 3). There was $5 \%$ mortality in the thrombolysed group compared to $17 \%$ in non-thrombolysed group in IWMI, suggesting that mortality was higher in non-thrombolysed group in IWMI. In RVMI also mortality was higher in non-thrombolysed (50\%) patients compared to thrombolysed patients (13\%). There was a drastic increase in mortality in patients of RVMI who present late after the onset of symptoms from nil presenting within 7 hours to $37.5 \%$ within 8 - 12 hours and $50 \%$ after 12 hours. Incidence of cardiogenic shock was much higher among patients with RVMI (16\%) as compared to (3\%) in patients without RVMI. There was doubling of incidence of AV blocks in RVMI patients (24\%) as compared to non-RVMI (12.3\%). There was doubling in the incidence of ventricular tachycardia in RVMI patients $(12 \%)$ as compared to without
RVMI (6\%). There was more than three times increase in incidence of hypotension in RVMI patients (40\%) as compared to non-RVMI patients (12.3\%) (Table 4).

\begin{tabular}{|c|c|c|}
\hline & $\begin{array}{l}\text { No. of IWMI } \\
\text { Patients }\end{array}$ & $\%$ \\
\hline $\begin{array}{c}\text { No. of Patients with } \\
\text { RVMI }\end{array}$ & 25 & $28 \%$ \\
\hline $\begin{array}{l}\text { No. of Patients } \\
\text { without RVMI }\end{array}$ & 65 & $72 \%$ \\
\hline Total Patients & 90 & $100 \%$ \\
\hline
\end{tabular}

In the above table among 90 patients of AC IWMI, 25 patients were found to have right ventricular infarction which accounts for $28 \%$ of incidence.

\begin{tabular}{|c|c|c|c|}
\hline & $\begin{array}{c}\text { Total } \\
\text { Patients }\end{array}$ & $\begin{array}{c}\text { Raised } \\
\text { JVP }\end{array}$ & $\mathbf{\%}$ \\
\hline RVMI & 25 & 15 & $60 \%$ \\
\hline $\begin{array}{c}\text { Without } \\
\text { RVMI }\end{array}$ & 65 & 20 & $30 \%$ \\
\hline $\begin{array}{c}\text { Total } \\
\text { Patients }\end{array}$ & $\mathbf{9 0}$ & $\mathbf{3 5}$ & $\mathbf{3 9 \%}$ \\
\hline \multicolumn{3}{|c|}{ Table 2. Jugular Venous Pressure } \\
\hline
\end{tabular}

$P=0.01$

In the above table, out of 25 patients with right ventricular involvement, 15 patients $(60 \%)$ had their JVP raised, whereas it was increased only in 20 patients out of 65 patients $(30 \%)$ of IWMI without right ventricular involvement.

\begin{tabular}{|c|c|c|c|}
\hline & $\begin{array}{c}\text { IWMI } \\
\text { Patients }\end{array}$ & Deaths & $\%$ \\
\hline RVMI & 25 & 4 & $16 \%$ \\
\hline $\begin{array}{c}\text { Without } \\
\text { RVMI }\end{array}$ & 65 & 2 & $3 \%$ \\
\hline $\begin{array}{c}\text { Total } \\
\text { Patients }\end{array}$ & $\mathbf{9 0}$ & $\mathbf{6}$ & $\mathbf{6 . 6 6 \%}$ \\
\hline \multicolumn{4}{|c|}{ Table 3. Mortality in IWMI Patients with and without } \\
RVMI
\end{tabular}

$\mathrm{P}=0.04$

In the above table, out of total mortality of 6 patients, 4 deaths occurred in RVMI group, which accounts for $16 \%$ mortality compared to 2 deaths in IWMI without RVMI group out of 65 patients, which is equal to $3 \%$ mortality in that group.

\begin{tabular}{|c|c|c|c|}
\hline & $\begin{array}{c}\text { IWMI } \\
\text { Patients }\end{array}$ & Hypotension & $\mathbf{\%}$ \\
\hline RVMI & 25 & 10 & $40 \%$ \\
\hline $\begin{array}{c}\text { Without } \\
\text { RVMI }\end{array}$ & 65 & 8 & $12.3 \%$ \\
\hline $\begin{array}{c}\text { Total } \\
\text { Patients }\end{array}$ & $\mathbf{9 0}$ & $\mathbf{1 8}$ & $\mathbf{2 0 \%}$ \\
\hline \multicolumn{4}{|c|}{ Table 4. Incidence of Hypotension in IWMI Patients with } \\
and without RVMI \\
\hline
\end{tabular}

$P=0.006$

In the above table, out of 25 patients with RVMI 10 patients had hypotension during their hospital stay, which is equal to $40 \%$ incidence, while only 8 patients in IWMI 
without RV involvement had hypotension accounting for $12.3 \%$ incidence.

\section{DISCUSSION}

In the present study entitled Incidence, clinical profile and inhospital outcome of patients of RVMI in IWMI, 90 patients with acute inferior wall MI who were admitted in Medicine ICCU Ward with chest pain duration of $<24$ hours were taken, because most ECG changes of acute myocardial infarction subsides within 24 hours. Patients who have history of COPD and with previous MI were not taken in this study, as these may act as confounding factors in the diagnosis of right-sided heart condition.

In the present study, most patients were in the age range of 41 - 80 years with slightly more patients falling in the age group of 41 - 60 years in both RVMI+ and RVMI- groups. This was not significant $P>0.05$, so this may be a chance association. This may be due to non-consecutive selection of patients. It shows that right ventricular myocardial infarction does not show any preponderance to a particular age group.

The mean age of our study population was 59.7 years, which correlated very well with the study of Mehta et al.13

In the present study population, there were 57 male patients (63\%) and 33 female patients (37\%). There were 13 patients with RVMI among 57 male patients of IWMI, which accounts for $22 \%$ incidence of RVMI in male patients. Of 33 female patients 12 were diagnosed to have right ventricular infarction, which accounts for 36\% incidence of RVMI in female population. Incidence of RVMI was found to be more in female population. Since $(p>0.05)$ this correlation was not significant, although increased incidence of RVMI was also reported by Mehta et al,13 but this was also statistically insignificant.

So, distribution of RVMI did not show any sexual predilection.

\section{Hypertension}

21 out of 90 patients of IWMI gave the history of hypertension, which accounts for $23 \%$ prevalence of hypertension. In the present study group among RVMI patients, 5 patients were hypertensive accounting for $20 \%$ prevalence in the RVMI group. Among 65 patients without RV involvement 16 patients were hypertensive leading to a prevalence of $24 \%$ in that group, but $\mathrm{P}$ value of this correlation was 0.7 indicating that this correlation might be by chance and no actual relationship between hypertension and increased incidence of RVMI exists.

However, Iqbal et al 15 found hypertension prevalent among $44 \%$ population of IWMI. However, this may be affected by different geographical, cultural and educational strata of the study population.

This shows that although hypertension is a well-known risk factor for coronary artery disease, it did not affect RV involvement.

\section{Diabetes Mellitus}

Type 2 diabetes mellitus was found in 27 out of total 90 patients of IWMI accounting for $30 \%$ incidence in the study population, whereas prevalence in RVMI group was 6 patients out of 25 patients (24\% prevalence) and prevalence in patients without RVMI was 21 out of 65 patients $(32 \%$ prevalence). But $\mathrm{P}>0.05$ in this association therefore appears to be no significant correlation between RV involvement and diabetes mellitus.

Similar results were found by Mehta et al,13 who found $18 \%$ prevalence of T2DM in IWMI patients with $16 \%$ in RVMI group, $15.9 \%$ among non-RVMI group.

\section{Risk Factors other than T2DM and Hypertension were as follows}

$40 \%$ patients were tobacco chewers and $29 \%$ were smokers, $9 \%$ patients were both smokers and tobacco chewers, $12 \%$ had history of alcohol abuse and 34\% had family history of CAD. All these are well-known risk factors for CAD and other vascular disease.

All these risk factors were distributed in both RVMI+ and RVMI- groups and were not found to be associated specifically with either of groups $p>0.05$.

\section{Presenting Complaints}

In the present study almost all patients presented with chest pain (100\%), $22 \%$ patients also complained of breathlessness, while $17.77 \%$ patients also had palpitations, $33 \%$ patients complained of perspiration and $10 \%$ patients had syncope or giddiness.

\section{Clinical Examination}

In the present study, JVP was found to be elevated in 35 patients of IWMI, out of which 15 had RVMI which accounts for $60 \%$ of patients with RVMI, while it was raised in only $30 \%$ patients without right ventricular involvement. Since P value of this observation $<0.05$, it is considered significant. So, it can be concluded that raised JVP is significantly associated with RVMI. However, in some other studies the percentage of raised JVP was found to be much higher. 16

Since it is a clinical parameter, subjective variation in the observation is likely. Nonetheless, elevated JVP remains strongly associated with RVMI.

Also, some studies have shown that since left ventricular function is also depressed secondary to RVMI, this along with conduction disorders associated with RVMI offsets the clinical manifestations of RVMI.

\section{Incidence of RVMI in Inferior Wall MI}

In the present study, RVMI was diagnosed in 25 patients out of 90 patients of IWMI.

Incidence of RVMI is variable depending upon the criteria used to diagnose it, so in many other studies its incidence varies from $10 \%$ to $50 \% \cdot 4,11,13,15$ In the present study we used ECG criteria as the sole method to diagnose RVMI, while most other studies have used echocardiography, right heart catheterisation and technetium scan to diagnose RVMI3,13,16 and because ST elevations in lead V4R mostly persists up to 12 hours after the MI, some patients are likely to be missed.

\section{Mortality in IWMI Patients}

In the present study, we followed the clinical course of 90 hospitalised IWMI patients. Out of 90 patients, we had 6 deaths leading to $7 \%$ mortality. Out of 6 deaths, 4 were diagnosed to have RVMI leading to mortality of $16 \%$ in RVMI group, whilst 2 deaths occurred in non-RVMI group leading to $3 \%$ mortality in that group.

There were 4 deaths among 25 RVMI patients $(16 \%$ mortality) and 2 deaths out of 65 patients without RVMI (3\% 
mortality). Mortality was significantly higher in RVMI group $\mathrm{P}$ $<0.05$.

Also in the study by Mehta et al ${ }^{13}$ mortality was higher in RVMI subgroup, $7.1 \%$ of IWMI compared to $5.4 \%$ in nonRVMI group.

Therefore, presence of right ventricular infarction in IWMI qualifies as high risk group. But mortality rate varies among different studies due to different management protocols and facilities available in different centres.

We divided IWMI patients according to whether they were thrombolysed or not. Out of 90 patients, 78 patients were thrombolysed including RV+ and RV- patients and 12 patients were treated without thrombolysis.

There were 4 deaths among thrombolysed patients leading to $5 \%$ mortality compared to $17 \%$ mortality in nonthrombolysed patients $(\mathrm{P}=0.15)$.

Similarly, patients with exclusively RVMI were divided in the thrombolysed and non-thrombolysed group, mortality was higher in non-thrombolysed sub-group (50\%) compared to $13 \%$ mortality in thrombolysed group.

In both IWMI group and RVMI group, mortality improved after thrombolysis more so in RVMI group. Many studies show improved outcome after successful thrombolysis. . $^{17,18,19,20,21,22,23}$

TIMI second phase also shows reduction in the incidence of mortality in RVI patients with successful thrombolysis. 19

Even though mortality improved with thrombolysis, in the present study it was statistically insignificant $\mathrm{P}>0.05$, but outcome improved. RVI.

Berger et $\mathrm{al}^{21}$ found thrombolytics reduces incidence of

$\mathrm{O}^{\prime}$ Rourke $^{22}$ recommends that patients with evidence of RVI by ECG criteria or by other non-invasive methods, who have clinical evidence of depressed right ventricular function are candidates for coronary angioplasty (if available), thrombolytic therapy (if not contraindicated).

\section{Chest Pain Duration}

In the present study, we classified our study cohort into 3 groups according to duration of chest pain; 48 patients out of 90 presented within 7 hours of onset of chest pain, 30 patients presented with chest pain duration of $8-10$ hours.

In patients presenting within 7 hours of onset of chest pain no death occurred, while 4 deaths occurred in the $8-12$ hours group accounting for $13.33 \%$ mortality. Out of 12 patients presenting with $12-24$ hours chest pain, 2 deaths occurred accounting for $16.66 \%$ mortality.

Similarly, in RVMI subgroup also, maximum no. of patients presented within initial 7 hours and had better prognosis compared to the late presenters with 3 deaths occurring in 8 - 12 hours subgroup accounting for $37.5 \%$ mortality, whilst of 2 patients presenting after 12 hours 1 death occurred.

In both IWMI and RVMI alone, prognosis was better in early presenters compared to late presenters and this difference was more prominent in the RVMI group and this was found to be significant $\mathrm{P}<0.05$ in the RVMI group.

In IWMI without RVMI too mortality was higher among late presenters with no mortality among early presenters and $4.5 \%$ mortality in patients with chest pain duration of $8-12$ hours and $10 \%$ mortality among patient presenting with more than 12 hours chest pain.
In the present study, early presenters had lower mortality which may be because of early treatment with thrombolysis and reperfusion of ischaemic myocardium and timely management of haemodynamic compromise. It may be influenced in case of RVMI by early recognition of RV involvement by electrocardiogram in the early hours of IWMI as ECG changes indicating right ventricular infarction mostly settle early within 12 hours.

\section{Complications \\ Cardiogenic Shock}

Out of 25 patients with RVMI 4 patients had cardiogenic shock accounting for $16 \%$ incidence in that group, while only 2 patients out of 65 patients had cardiogenic shock (3\%). This was significant $(\mathrm{P}=0.04)$.

Mehta et al ${ }^{13}$ also found $6.9 \%$ incidence of shock in RVI patients compared to $5.5 \%$ incidence without RVMI.

AV blocks: In the present study, we evaluated the incidence of $2^{\circ}$ and higher blocks in patients with and without RVMI. Out of 25 patients with RVMI 6 had AV blocks, which is $24 \%$ incidence in that group, whilst 8 patients of IWMI without RVMI had AV blocks accounting 12.3\% incidence. So incidence of AV blocks was found higher in RVMI group.

$\mathrm{P}=0.01$, therefore there is significant association between AV blocks and incidence of RVMI.

Mehta et $\mathrm{al}^{13}$ also showed similar results in their study with $21 \%$ incidence in RVMI group compared to $9 \%$ incidence in non-RVMI group.

Bueno et al ${ }^{14}$ in 1998 found AV blocks in $25 \%$ of patients of RVMI and $6.7 \%$ patient of IWMI without RVMI.

Prevention and management of AV blocks and bradyarrhythmia are very important, because they cause AV dyssynchrony leading to loss of atrial contribution to ventricular filling further jeopardising already decreased cardiac output.

\section{Ventricular Tachycardia}

Out of 25 patients with RVMI 3 had Ventricular Tachycardia which is $12 \%$ incidence in that group, whilst 4 patients of IWMI without RVMI had Ventricular Tachycardia accounting for $6 \%$ incidence. So, incidence of VT was found to be higher in RVMI group. Although $\mathrm{p}>0.05$, incidence was found to be twice that of patients without RVMI

Mehta et al ${ }^{13}$ also found increased incidence of VT/VF (Three times) in patients with RVMI. They attributed increased mortality in RVMI group to increased arrhythmias.

Berger et $\mathrm{al}^{21}$ reported $8.6 \%$ incidence in RVMI group compared to $2.5 \%$ in non-RVMI group.

Hypotension - It is one of clinical hallmark of RVMI. Out of 25 patients with RVMI 10 patients had hypotension during their hospital stay, which is equal to $40 \%$ incidence, whilst only 8 patients in IWMI without RV involvement had hypotension accounting for $12.3 \%$ incidence $\mathrm{P}=0.006$, which was very significant. Therefore, hypotension was much more common in patients with RVMI.

Mehta et $\mathrm{al}^{13}$ reported hypotension in $29 \%$ patients with RVMI compared to $19.3 \%$ in patients of IWMI without RV involvement.

Hypotension - In RVMI occurs due to impaired filling of left ventricle secondary to impaired right ventricular cardiac output and decreased LV filling pressures, therefore volume 
loading in case of RVMI is an important therapeutic approach to prevent cardiogenic shock.

\section{CONCLUSION}

Right ventricular myocardial infarction occurs in a significant number of patients with inferior wall myocardial infarction. Extent of right ventricular involvement varies from patient to patient and ranges from myocardial stunning to full blown infarction.

It represents a high risk subgroup of IWMI with distinct clinical presentation comprising of raised JVP and hypotension with clear lung fields, which requires a high degree of clinical suspicion.

However, it can be easily diagnosed with considerable accuracy by a simple bedside routine 12-lead ECG along with right-sided ECG leads. Since ECG changes are transient in right-sided ECG, it is difficult to diagnose RVMI after 12 hours. So, ECG should be supplemented by urgent routine echocardiography to improve diagnostic yield.

Frequent accompaniments of RVMI include atrial infarction, sinus bradycardia, ventricular tachycardia, atrial fibrillation, atrioventricular block and cardiogenic shock. Patients with RVI who are haemodynamically unstable should be managed with volume loading to maintain adequate right ventricular preload. Early recognition and prompt reperfusion with thrombolytic therapy or coronary angioplasty, rate and rhythm control and inotropic support may also be warranted since patients with RV infarction have high incidence of complications and in-hospital mortality.

Patients who survive the acute phase have no long-term consequences and complete recovery over a period of weeks to months is the rule in majority of patients, suggesting right ventricular "stunning" rather than irreversible necrosis.

Therefore, right-sided ECG should be taken routinely in all patients of inferior wall MI.

\section{REFERENCES}

[1] Cohn JN, Guiha NH, Broder MI, et al. Right ventricular infarction. Clinical and hemodynamic features. Am J Cardiol 1974;33(2):209-14.

[2] Menown IB, Allen J, Anderson JM, et al. Early diagnosis of right ventricular or posterior infarction associated with inferior wall left ventricular acute myocardial infarction. Am J Cardiol 2000;85(8):934-8.

[3] Kinch JW, Ryan TJ. Right ventricular infarction. N Engl J Med 1994;330(17):1211-7.

[4] Khan S, Kundi A, Sharieff S. Prevalence of right ventricular myocardial infarction in patients with acute inferior wall myocardial infarction. International Journal of Clinical Practice 2004;58(4):354-7.

[5] Anderson HR, Falk E, Nielson D. Right ventricular infarction: frequency, size and topography in coronary heart disease: a prospective study comprising 107 consecutive autopsies from a coronary care unit. J Am Coll Cardiol 1987;10(6):1223-32.

[6] Brookes C, Ravn H, White $\mathrm{P}$, et al. Acute right ventricular dilatation in response to ischemia significantly impairs left ventricular systolic performance. Circularion 1999;100(7):761-7.
[7] Goto Y, Yamamoto J, Saito M, et al. Effects of right ventricular ischemia on left ventricular geometry and the end-diastolic pressure-volume relationship in the dog. Circulation 1985;72:1104-14.

[8] Goldstein JA. Pathophysiology and management of right heart ischemia. J Am Coll Cardiol 2002;40(5):841-53.

[9] Braat SH, Gorgels AP, Bär FW, et al. Value of the ST-T segment in lead V4R in inferior wall acute myocardial infarction to predict the site of coronary arterial occlusion. Am J Cardiol 1988;62(1):140-2.

[10] Klein HO, Tordjman T, Ninio R, et al. The early recognition of right ventricular infarction: diagnostic accuracy of the electrocardiographic V4R lead. Circulation 1983;67:558-65.

[11] Showkaat HA, Movahed A. Right ventricular infarctiondiagnosis and treatment. Clin Cardiol 2000;23:473-82.

[12] Carter T, Ellis K. Right ventricular infarction. Crit Care Nurse 2005;25(2):52-4, 56-8, 60-2.

[13] Mehta SR, Eikelboom JW, Natarajan MK, et al. Impact of right ventricular involvement on mortality and morbidity in patients with inferior myocardial infarction. Journal of the American College of Cardiology 2001;37(1).

[14] Bueno H, López-Palop R, Bermejo J, et al. In-hospital outcome of elderly patients with acute inferior myocardial infarction and right ventricular involvement. Circulation 1997;96(2):436-41.

[15] Iqbal A, Muddarangappa R, Shah SKD, et al. A study of right ventricular infarction in inferior wall myocardial infarction. J Clin Sci Res 2013;2:66-71.

[16] Dell'Italia LJ, Starling MR, O'Rourke RA. Physical examination for exclusion of hemodynamically important right ventricular infarction. Ann Intem Med 1983;99(5):608-11.

[17] Zehender M, Kasper W, Kauder E, et al. Eligibility for and benefit of thrombolytic therapy in inferior myocardial infarction: focus on the prognostic importance of right ventricular infarction. J Am Coll Cardiol 1994;24(2):362-9.

[18] Kinn JW, Ajluni SC, Samyn JG, et al. Rapid hemodynamic improvement after reperfusion during right ventricular infarction. J Am Coll Cardiol 1995;26(5):1230-4.

[19] Gumina RJ, Wright RS, Kopecky SL, et al. Strong predictive value of TIMI risk score analysis for inhospital and long-term survival of patients with right ventricular infarction. Eur Heart J 2002;23(21):167883.

[20] Bueno H, López-Palop R, Pérez-David E, et al. Combined effect of age and right ventricular involvement on acute inferior myocardial infarction prognosis. Circulation 1998;98(17):1714-20.

[21] Berger PB, Ruocco NA, Ryan TJ, et al. Frequency and significance of right ventricular dysfunction during inferior wall left ventricular myocardial infarction treated with thrombolytic therapy (results from the thrombolysis in myocardial infarction [TIMI] II trial). The TIMI research group. Am J Cardiol 1993;71(13):1148-52. 


\section{Jemds.com}

[22] O'Rourke RA. Treatment of right ventricular infarction: thrombolytic therapy, coronary angioplasty or neither. J Am Coll Cardiol 1998;32(4):882-4.
Original Research Article

[23] Zeymer U, Neuhaus KL, Wegscheider K, et al. Effects of thrombolytic therapy in acute inferior myocardial infarction with or without right ventricular involvement. HIT-4 trial group. Hirudin for improvement of thrombolysis. J Am Coll Cardiol 1998;32(4):876-81. 